\title{
Editorial
}

\section{Advances on the Resilience of Complex Networks}

\author{
Ilaria Giannoccaro $\mathbb{D}^{1}{ }^{1}$ Vito Albino, ${ }^{1}$ and Anand Nair ${ }^{2}$ \\ ${ }^{1}$ Department of Mechanics, Mathematics, and Management, Polytechnic University of Bari, Bari, Italy \\ ${ }^{2}$ Department of Supply Chain Management, Eli Broad College of Business, Michigan State University, East Lansing, MI, USA
}

Correspondence should be addressed to Ilaria Giannoccaro; ilaria.giannoccaro@poliba.it

Received 26 July 2018; Accepted 26 July 2018; Published 13 August 2018

Copyright ( 2018 Ilaria Giannoccaro et al. This is an open access article distributed under the Creative Commons Attribution License, which permits unrestricted use, distribution, and reproduction in any medium, provided the original work is properly cited.

A common property of many complex systems is resilience, that is, the ability of the system to react to perturbations, internal failures, and environmental events by absorbing the disturbance and/or rebuild to maintain its functions. Nowadays, understanding how complex systems demonstrate resilience is critical in many different fields, because examples of collapses and crises caused by low resilience are more and more spreading all over the world including transportation, financial, energy, communication, and ecological systems.

Therefore, in the last decade, the topic of resilience has grown a lot in popularity. Studies on resilience are popular in multiple disciplines, such as ecology, environmental science, computer science, engineering, management science, economics, and phycology. They investigate resilience of a broad variety of complex systems involving individuals, teams, ecosystems, organizations, communities, supply chains, financial networks, computer networks, and building infrastructures.

Despite this multidisciplinary nature, two main perspectives in the conceptualization of resilience are recognized, that is, the static and dynamic ones [1-4]. The resilience is static when it focuses on the ability of the system to absorb disturbance and bounce back to the original equilibrium state, maintaining its core functions when shocked. In such a case, the resilience is linked to the ability to recover the original shape and features once stretched (robustness) and the capacity of the system to take alternative positions to respond better to change (flexibility). The dynamic perspective focuses on the ability of the system to evolve over time moving towards a new more favorable equilibrium state.
According to this perspective, resilience concerns the adaptive capacity of the system, which is able to react to disturbance by changing its structure, processes, and functions in order to increase its ability to persist [5].

This special issue collects nine papers concerning resilience of complex systems, which accords well with the main features summarized above. They concern studies investigating resilience of complex systems in diverse disciplines (engineering, management science, computer science, economics, and organization science) and adopting both the static and dynamic perspectives. Their aim is to identify the main factors and dynamics influencing resilience of diverse systems (water system infrastructures, organizational teams, financial markets, wireless sensor network, and urban system) to a variety of unexpected and negative events.

First, the paper by L. Fraccascia et al. provides an updated state of the art on the resilience of complex systems using bibliometric tools. The review is carried out embracing the multiple research areas, with the aim of analyzing how resilience is approached in multiple fields, how the topic evolved starting from the ecological field of study, and the level of cross-fertilization among domains. The authors also identify main gaps of the current literature and provide some directions for future research. In this regard, they suggest that network theory and its measures can play a relevant role to model complex systems and investigate the relationship between network topology and resilience.

Two papers of the special issue precisely follow this suggestion and apply network theory to model the complex system and analyze resilience using network measures. In particular, the paper by M. Cinelli et al. entitled "Resilience 
of Core-Periphery Networks in the Case of Rich-Club" uses core-periphery network to investigate the rich-club phenomenon and how it relates to resilience. They measure resilience of different network structures, characterized by progressively denser rich-club obtained by adding links to the core and the periphery, and discuss effects on network cohesion and efficiency.

A. Pagano et al. in the paper entitled "Integrating "Hard" and "Soft" Infrastructural Resilience Assessment for Water Distribution Systems" develop an integrated approach, based on graph theory and social network analysis, to assess resilience of infrastructural systems with specific reference to water distribution systems. The urban system is modelled as a set of interrelated and evolving networks including the "hard" infrastructural system (i.e., the water distribution system) and the "soft" infrastructure (i.e., the social network for emergency management). The methodology proposed is applied to measure resilience of the hard/soft infrastructures during the L'Aquila 2009 earthquake.

In the paper by Y. Wang et al. entitled "Impact of Rapid Urbanization on Vulnerability of Land System from Complex Networks View: A Methodological Approach," network measures are coupled with a simulation approach. In particular, the authors develop a methodological approach by integrating complex network model and disruptive scenarios to explore the effects of urbanization on land system structural vulnerability. They apply their model to Wuhan City in China and find that the land system is more vulnerable to intentional attacks on nodes with higher integrated node centrality and larger land area and that the network efficiency of the land system strongly depends on shrinkage rate of land use types.

While the studies above deal with resilience of engineering and urban systems, the paper entitled "Analysis on Invulnerability of Wireless Sensor Network towards Cascading Failures Based on Coupled Map Lattice" by X. $\mathrm{Fu}$ et al. addresses this issue in the field of computer science. This study extends previous research on wireless sensor network vulnerability with respect to cascading failures based on the coupled map lattice. Network topological issues are also investigated with the aim of designing robustness networks. In particular, four types of network topologies (random network, small-world network, homogenous scale-free network, and heterogeneous scale-free network) under various attack schemes are compared and the results are discussed.

A common feature of all these studies above is that they analyze resilience according to a static perspective, focusing on the properties of vulnerability and robustness under attacks. The following three papers of the special issue adopt the dynamic perspective and model resilience as an emergent property of the complex system by using simulation approaches.

The paper by G. F. Massari et al. entitled "Team Resilience in Complex and Turbulent Environments: The Effect of Size and Density of Social Interactions" studies team resilience as the ability of the team as a whole to adapt to change and react to the disturbance by reaching the same or better performance than those before the disruption. They employ a model of team decision-making where collective dynamics of team members are governed by a continuous-time Markov process. Two drivers of team resilience are investigated: the team size and the density of social interactions among team members.

In the paper by K. Mahmoodi et al. entitled "SelfOrganized Temporal Criticality: Bottom-Up Resilience versus Top-Down Vulnerability," the authors propose a physical statistic model of spontaneous self-organization, generating criticality and resilience. Two approaches to self-organized criticality are analyzed: (1) the spontaneous bottom-up emergence of cooperation and altruism and (2) the top-down process mimicking the leadership of an elite. They show with numerical simulation that while both models generate criticality, only one, that is, the bottom-up approach, determines resilience. Implications for the organization of social systems are also discussed.

The study by L. Ponta and S. Cincotti entitled "Traders' Networks of Interactions and Structural Properties of Financial Markets: An Agent-Based Approach" investigates the resilience of financial stock markets using an agent-based simulation approach. They develop an information-based multiasset artificial stock market characterized by different types of stocks and populated by heterogeneous agents and investigate the impact of the structural properties of traders' networks of interaction on the emergent outcome of the financial system. Their results show the intrinsic structural resilience of the stock market.

\section{Conflicts of Interest}

The editors declare that they have no conflicts of interest regarding the publication of the special issue.

\section{Acknowledgments}

The guest editorial team would like to express gratitude to all the authors for their interest in selecting this special issue as a venue for disseminating their scholarly work. The editors also wish to thank the anonymous reviewers for their careful reading of the manuscripts submitted to this special issue collection and their many insightful comments and suggestions.

\section{Ilaria Giannoccaro Vito Albino Anand Nair}

\section{References}

[1] A. Rose, "Defining and measuring economic resilience to disasters," Disaster Prevention and Management: An International Journal, vol. 13, no. 4, pp. 307-314, 2004.

[2] A. Annarelli and F. Nonino, "Strategic and operational management of organizational resilience: current state of research and future directions," Omega, vol. 62, pp. 1-18, 2016.

[3] C. A. Lengnick-Hall, T. E. Beck, and M. L. Lengnick-Hall, "Developing a capacity for organizational resilience through strategic human resource management," Human Resource Management Review, vol. 21, no. 3, pp. 243-255, 2011. 
[4] L. Fraccascia, I. Giannoccaro, and V. Albino, "Rethinking resilience in industrial symbiosis: conceptualization and measurements," Ecological Economics, vol. 137, pp. 148-162, 2017.

[5] B. Smit and J. Wandel, "Adaptation, adaptive capacity and vulnerability," Global Environmental Change, vol. 16, no. 3, pp. 282-292, 2006. 


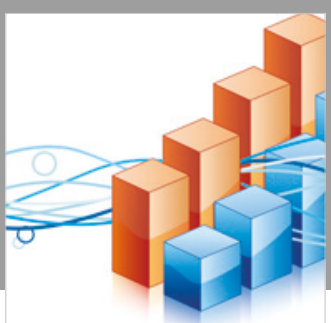

Advances in

Operations Research

\section{-n-m}
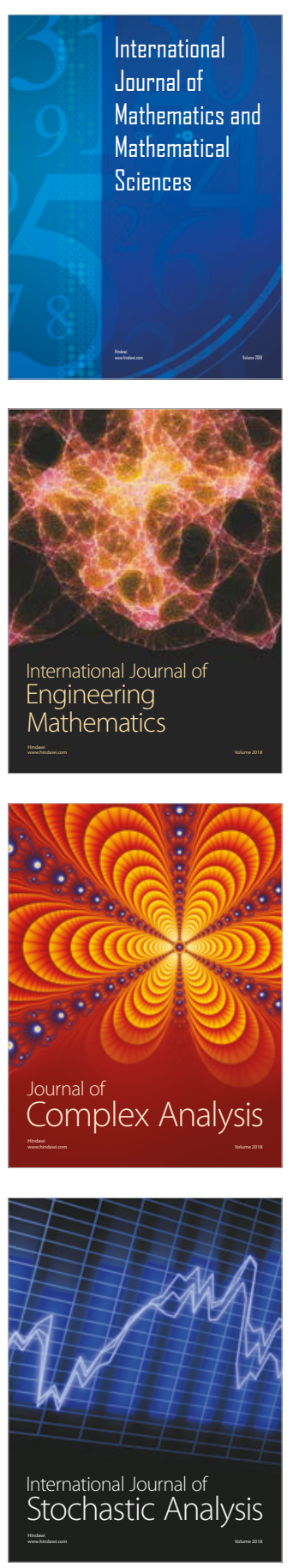
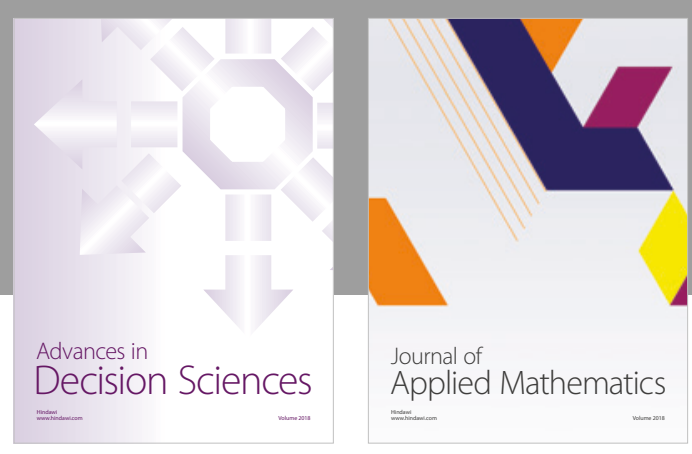

Journal of

Applied Mathematics
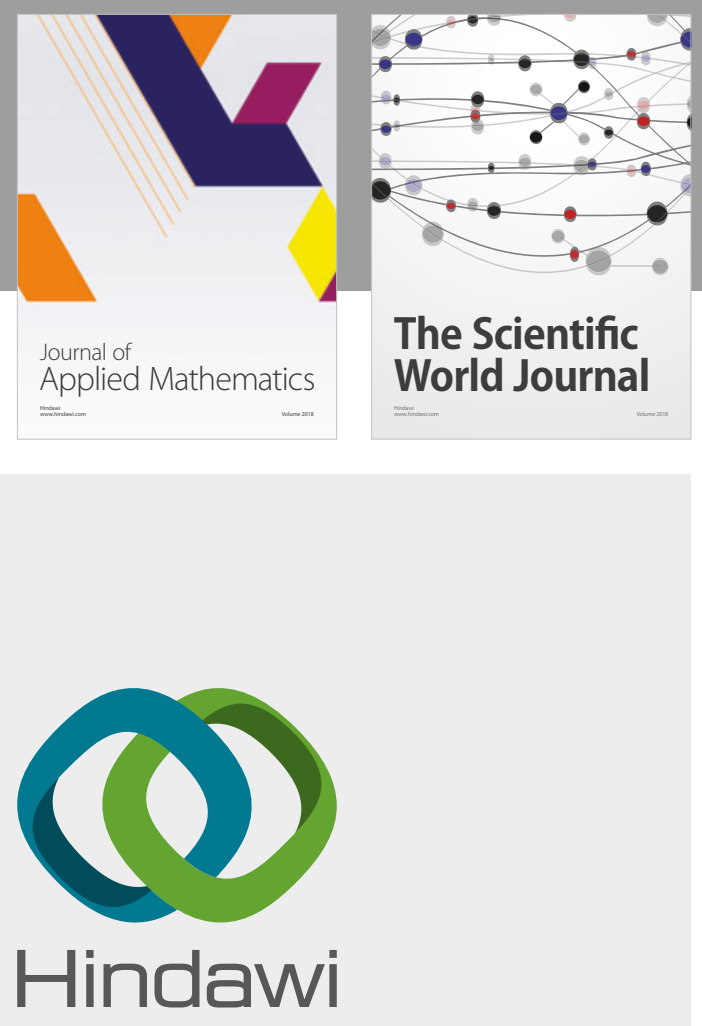

Submit your manuscripts at

www.hindawi.com

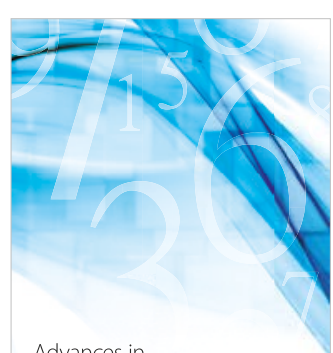

Advances in
Numerical Analysis
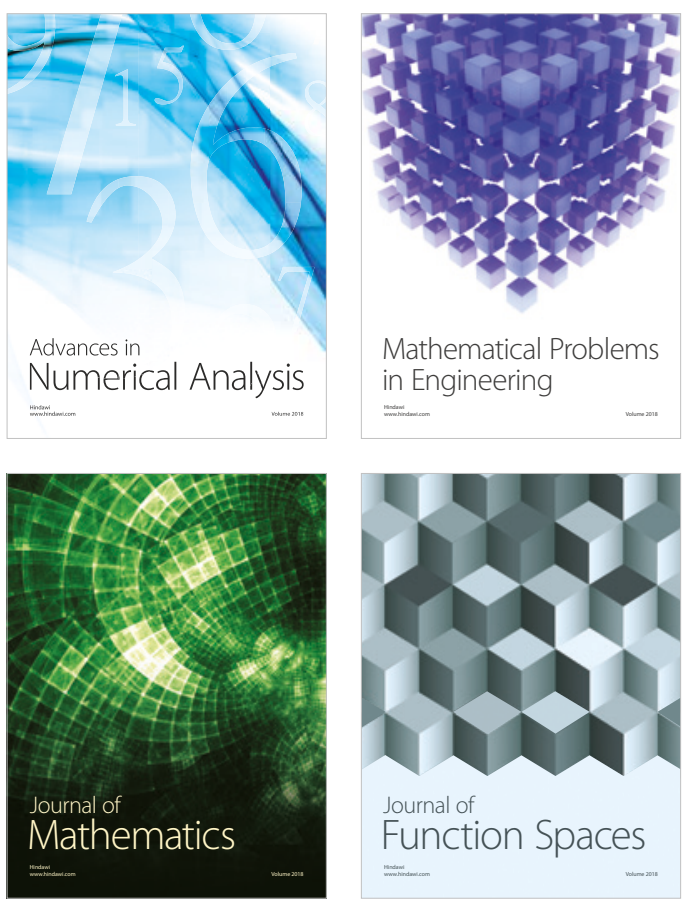

Mathematical Problems in Engineering

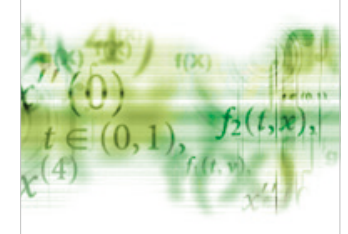

International Journal of

Differential Equations

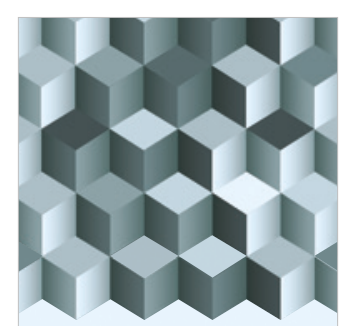

Journal of

Function Spaces
The Scientific

World Journal

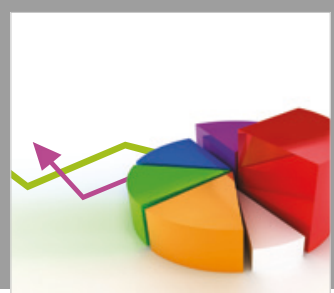

Journal of

Probability and Statistics
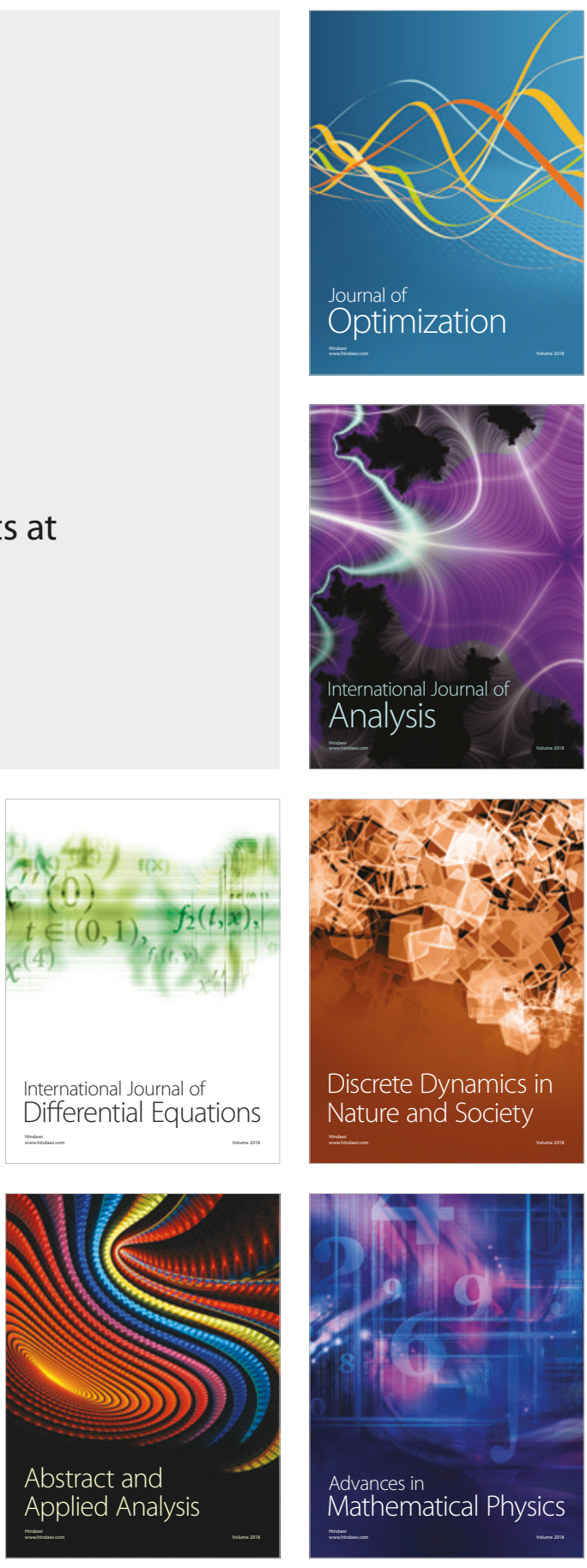